\title{
Mechanical Factors Influencing Bending of Pole in Pole Vault
}

\author{
Takafumi Kageyuki ${ }^{1,2}$, Takeo Matsubayashi ${ }^{3}$, Tomoki Yamamoto ${ }^{4}$, Fumiaki Kobayashi ${ }^{4}$, \\ Takeshi Asai ${ }^{5}$, Keigo Ohyama-Byun ${ }^{5}$, and Kiyonobu Kigoshi ${ }^{5}$ \\ ${ }^{1}$ Graduate School of Comprehensive Human Sciences, University of Tsukuba, \\ 1-1-1 Tennodai, Tsukuba, Ibaraki 305-8574 Japan \\ Takafumi Kageyuki (zkiac10@gmail.com) \\ ${ }^{2}$ Ibaraki Christian University, 6-11-1 Omikacho, Hitachi, Ibaraki 319-1295 Japan \\ ${ }^{3}$ Japan Institute of Sports Sciences, 3-15-1 Nishigaoka, Kita-ku, Tokyo 115-0056 Japan \\ ${ }^{4}$ Faculty of Sport Science, Nippon Sport Science University, \\ 1221-1 Kamoshidacho Aoba-Ku, Yokohama, Kanagawa 227-0033 Japan \\ ${ }^{5}$ Faculty of Health and Sport Sciences, University of Tsukuba, \\ 1-1-1 Tennodai, Tsukuba, Ibaraki 305-8574 Japan
}

[Received February 11, 2020; Accepted June 14, 2020; Published online July 8, 2020]

This study aimed to determine the best indicator to evaluate the ability to bend the pole and to clarify the mechanical factors that can influence pole bending with the run-up and pole in the same settings as those in competitions. Eight male vaulters $(4.60 \mathrm{~m}$ to $5.77 \mathrm{~m}$ : personal best record) participated. Three-dimensional body coordinates were obtained using a motion capture system $(250 \mathrm{~Hz})$. In addition, the box reaction force was measured with a force plate $(1000 \mathrm{~Hz})$. The results were summarized as follows:

(1)The strain elastic energy in the pole $\left(E_{\text {pole }}\right)$ at maximal pole bending (MPB) showed higher correlation with the maximal height of the vaulter's center of gravity (CG) than the maximal bending rate and the maximal amount of pole bending $(r=0.94, r=0.86, r=0.87$, respectively).

(2)The larger the decrease in the translational energy and the smaller the increase in the potential energy in pole bending phase, the higher the $E_{p o l e}$ at MPB $(r=-0.87$ and -0.80 , respectively).

(3)The larger the moment of inertia around the CG and the upper grip at the instant where the negative peak value of the angular momentum around the CG appeared, the higher the $E_{\text {pole }}$ at MPB ( $\mathrm{r}=0.75$ and 0.79 , respectively).

These results suggested that the $E_{p o l e}$ was a more desirable indicator to evaluate the ability to bend the pole. Additionally, these results can serve as a basis for the practical application of techniques, namely, the swing motion with a fully extended posture.

Keywords: Athletics, motion capture system, energy, technique

\section{Introduction}

Pole vault is an athletic event wherein the participants compete to vault the greatest height over a crossbar with the aid of a pole. Hay (1993) analyzed the final vault height based on several factors; the increase in the height of the vaulter's center of gravity (CG) while being on the pole made the largest relative contribution to the final height of the pole vault. In addition, previous studies showed that the grip height, namely, the distance between the lower tip of the pole and the upper grip, had a significant effect on the vaulter's performance (Angulo-Kinzler et al., 1994; McGinnis, 1987). These imply that the grip height is a relevant factor in maximizing the height of the CG when the pole can be brought to a vertical position. The pole may be composed of either a single material or a combination of materials and has various lengths and diameters; however, its surface must be smooth (International Association of Athletics Federations, 2017). Elastic poles are most commonly used because vaulters can use higher grip point compared with inelastic poles (Hay, 1993). Bending the pole reduces 
the moment of inertia of the vaulter-pole system around the lower tip of the pole, thereby making it easier for the whole system to rotate around the lower tip of the pole. Therefore, the availability of a higher grip can be facilitated by bending the pole.

Previous studies used the bending rate (the amount of pole bending in relation the grip height) and the length of the pole chord (the distance between the lower tip of the pole and the upper grip during the vault) to evaluate the ability to bend the pole (Gros and Kunkel, 1990; Gudelj et al., 2015; Schade et al., 2004b). However, different vaulting poles can have different lengths and stiffness for the same bending rate, the absolute amount of pole bending varies depending on the length of the pole. Furthermore, the force and moment necessary for the same amount of bending depend on the stiffness of the pole. Therefore, the strain elastic energy: a variable that includes the absolute amount of bending and stiffness of the pole, would be a more desirable indicator to evaluate the ability to bend the pole. However, the influence of the strain elastic energy on the maximal height of the CG has not been studied.

The energetic interaction between the vaulter and pole has been clarified in previous studies (Dillman and Nelson, 1968; Hay, 1971; Arampatzis et al., 2004; Schade et al., 2000, 2004a, 2006; Schade et al., 2004b; Schade and Brüggemann, 2006; Sheehan, 2002). Accordingly, the vaulter's mechanical energy obtained in the run-up is transferred to the pole and stored as strain energy. However, the detailed interaction, namely, the influence of the change in the kinetic and potential energy on the strain elastic energy in the pole, has not been discussed.

The pole vault has been referred to as a double pendulum of the vaulter and pole (Dyson, 1977; Hay, 1993). Thus, there is a possibility that the vaulter's motion and the behaviour of the pole interact with each other. For instance, the effectiveness and importance of the "penetration" and "swing" for pole bending has been recognized practically (Hay, 1993; Petrov, 2004). The penetration is the generic term for the follow-through motion after take-off (TO), while the swing is the angular motion around grips to shift from the penetration to the rock back position. However, the effectiveness of these vaulting motions has not been discussed in detail or clarified biomechanically.

Meanwhile, the use of a less rigid pole could affect the motion and performance in pole vault (Frère et al., 2012a; Linthorne and Weetman, 2012). These studies have implied that vaulters could not vault in a similar way as in competitions in the trials with fewer run-up steps. However, fewer run-up steps and a less rigid pole during competitions have been adopted in previous studies (Arampatzis et al., 2004; Frère et al., 2012a; Schade et al., 2006; Schade and Arampatzis, 2012).

Therefore, this study aimed to determine the desirable indicator to evaluate the ability to bend the pole and to clarify the mechanical factors that can influence pole bending with the run-up and pole in the same setting as those during competitions. Thus, it was hypothesized that the strain elastic energy in the pole was a more relevant indicator to evaluate the pole bending than the bending rate and the amount of bending. In addition, it was hypothesized that the vaulter's motion, such as the penetration and the vigorous swing, generated a larger pole bending.

\section{Methods}

\subsection{Participants}

Eight experienced Japanese male vaulters (mass = $69.6 \pm 4.5 \mathrm{~kg}$; height $=1.78 \pm 0.04 \mathrm{~m}$; season best vault height $=5.32 \pm 0.36 \mathrm{~m}$ ), from the intercollegiate entry level to the top international level, participated in this study. This experiment was carried out as an applied research project for Athletics, managed by the Department of Sports Sciences, Japan Institute of Sports Sciences, and as a part of the activities of high performance strategy conducted by the Japan Association of Athletics Federations. In addition, this experiment was approved by the Ethics Committee of the Japan Institute of Sports Sciences.

\subsection{Experimental procedures}

After individual warm-up, the participants undertook trials using run-up steps and poles identical to those in competitions. The height of the bar (made of rubber; height $=5.05 \pm 0.23 \mathrm{~m}$ ) for each trial was determined by the participants, who were instructed to vault over the bar as high as possible. After the motion from the instant of touchdown of the take-off foot (TD) to the instant at which the peak height of the CG appeared (HP) was recorded, that from TO to pole straight (PS) was analyzed (Frère et al., 2010 ; Frère et al., 2012b). The trial that recorded the highest max- 
imal height of the CG on each participant was analyzed. Maximal pole bending (MPB) was defined as the instant at which the bending rate reached its maximum; PS was defined as the instant at which the pole was fully extended. Additionally, the instant that represented the swing motion has been defined based on the angular momentum in previous study (Angulo-Kinzler et al., 1994). Therefore, to represent the swing motion, SW was defined as the instant at which the angular momentum around the $\mathrm{CG}$ reached the negative peak value between TO and MPB.

\subsection{Data collection and processing}

The experiments were performed on an indoor experimental field. The global coordinate system was defined as the Y-axis in the opposite direction to the progress of the run-up, the Z-axis in the vertical upward direction, and the $\mathrm{X}$-axis as the cross product of the Y-axis and Z-axis. 37 reflective markers were attached to specific body landmarks based on the Plugin Gait protocols. The three-dimensional coordinates of the reflective markers were obtained using a motion capture system (Vicon Motion System Ltd., Oxford, UK; three MX Giganets, four T40 cameras, 16 T20 cameras, and four T10 cameras) and analyzed using the Nexus software (Vicon Motion System Ltd., Oxford, UK). All cameras were operated at $250 \mathrm{~Hz}$. The obtained three-dimensional coordinates were smoothed using a fourth-order Butterworth digital filter at optimum cutoff frequencies $(15-20 \mathrm{~Hz})$, which were determined using residual analysis methods (Wells and Winter, 1980). The vaulter's body was constructed using a 15-segment model (Winter, 1990) from the three-dimensional coordinates. In addition, the box re- action force acting on the lower tip of the pole was measured with a force plate (Kistler Instrument AG, Winterthur, Switzerland; 9287C, $0.9 \mathrm{~m} \times 0.6 \mathrm{~m}$, sampled at $1000 \mathrm{~Hz}$ ). The signal from the force plate was synchronized with the motion capture system using an eight-channel charge amplifier (Kistler Instrument AG, Winterthur, Switzerland; 9865E1Y28). The obtained box reaction force was smoothed using a fourth-order Butterworth digital filter with cutoff frequencies of 20 Hz.

\subsection{Data analysis}

The CG was calculated based on the inertial parameters of the body segment (Winter, 1990), and the CG velocity was calculated by differentiating the CG according to time. The grip height was defined as the distance between the lower tip of the pole and the upper grip at PS, while the pole chord was defined as the line segment connecting the lower tip of the pole to the upper grip during pole bending. However, the coordinates of the reflective marker attached on the $2^{\text {nd }}$ metacarpal bone head on the upper grip side was defined as the upper grip. The amount of pole bending $\left(A_{P B}\right)$ was defined as the difference between the grip height and the length of the pole chord. The bending rate was calculated from the $A_{P B}$ based on the grip height.

The mechanical energy of the vaulter $\left(E_{m}\right)$ was calculated as follows (Figure 1):

$$
\begin{aligned}
E_{m} & =E_{p}+E_{t}+E_{r}=\sum_{i=1}^{15}\left(E_{p_{i}}+E_{t_{i}}+E_{r_{i}}\right) \\
& =\sum_{i=1}^{15}\left(m_{i} g h_{i}+\frac{1}{2} m_{i} \boldsymbol{v}_{i}^{2}+\frac{1}{2} I_{i} \boldsymbol{\omega}_{i}^{2}\right)
\end{aligned}
$$

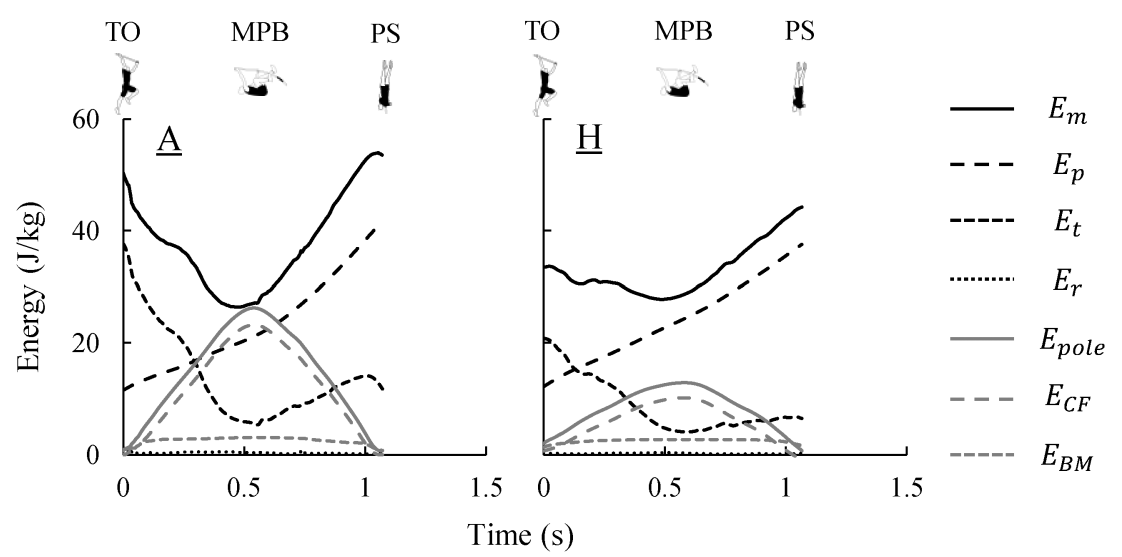

Figure 1 Mechanical energy of vaulter and strain elastic energy in the pole during the pole vault $\mathrm{A}$ and $\mathrm{H}$ indicate participants who recorded the highest and lowest maximal height of the CG, respectively 
where $E_{p}$ is the potential energy of the vaulter, $E_{t}$ is the translational kinetic energy of the vaulter, $E_{r}$ is the rotational kinetic energy of the vaulter, $E_{p_{i}}$ is the potential energy of the $i^{\text {th }}$ segment, $E_{t_{i}}$ is the translational kinetic energy of the $i^{\text {th }}$ segment, $E_{r_{i}}$ is the rotational kinetic energy of the $i^{\text {th }}$ segment, $m_{i}$ is the mass of the $i^{\text {th }}$ segment, $g$ is the gravitational acceleration, $h_{i}$ is the vertical component of the center of the mass coordinates of the $i^{\text {th }}$ segment in relation to the ground, $\boldsymbol{v}_{i}$ is the velocity vector of the $i^{\text {th }}$ segment, $\boldsymbol{I}_{i}$ is the moment of inertia of the $i^{\text {th }}$ segment, and $\boldsymbol{\omega}_{i}$ is the angular velocity vector of the $i^{\text {th }}$ segment rotated around the center of the mass of the $i^{\text {th }}$ segment.

The amount of the change in $E_{m}, E_{p}, E_{t}$, and $E_{r}$ from TO to MPB was defined as $\Delta E_{m}, \Delta E_{p}, \Delta E_{t}$, and $\Delta E_{r}$, respectively.

The strain elastic energy in the pole $\left(E_{\text {pole }}\right)$ was calculated as follows (Arampatzis et al., 2004):

$$
\begin{aligned}
& E_{\text {pole }}=E_{C F}+E_{E M} \\
& E_{C F}=\int C F d A_{P B} \\
& E_{B M}=\int B M d \theta_{P B}
\end{aligned}
$$

where $E_{C F}$ and $E_{B M}$ were the strain elastic energy derived from compression force $(C F)$ and bending moment $(B M)$, respectively; $C F$ is the force that projected the box reaction force on the pole chord; $B M$ is the bending moment applied to the upper end of the pole, which is calculated as the cross product between the vector from the lower tip of the pole to the upper grip and the force projected the box reaction force on the plane defined by the lower tip of the pole and both grips; and $\theta_{P B}$ is the angle between the pole chord and the line segment connecting both grips. However, energetic variables were normalized by the vaulter's body mass.

The angular momentum around the CG and the upper grip were calculated as follows:

$$
\boldsymbol{L}=\sum_{i=1}^{15} m_{i}\left(\boldsymbol{r}_{i}^{\prime} \times \boldsymbol{v}_{i}^{\prime}\right)+\sum_{i=1}^{15} \boldsymbol{I}_{i} \boldsymbol{\omega}_{i}
$$

where $\boldsymbol{r}_{i}^{\prime}$ is the position vector of the $i^{\text {th }}$ segment in the relative coordinate system with the CG and the upper grip as the origin and $\boldsymbol{v}_{i}^{\prime}$ is the velocity vector of the $i^{\text {th }}$ segment in the relative coordinate system. In addition, the moment of inertia around the CG and the upper grip was calculated as follows:

$$
\boldsymbol{I}=\sum_{i=1}^{15}\left(\boldsymbol{I}_{i}+m_{i} \boldsymbol{r}_{i}^{\prime 2}\right)
$$

However, kinetic variables within only Y-Z plane were calculated because the $\mathrm{Y}$ and $\mathrm{Z}$ components of the box reaction force in this study were far larger than the $\mathrm{X}$ component reported in previous studies (Arampatzis et al., 2004; Schade et al., 2006); it has been clarified that the angular momentum around the CG within $\mathrm{Y}-\mathrm{Z}$ plane was higher than the two other planes (Morlier and Cid, 1996). In addition, the angular momentum and the moment of inertia were normalized by the vaulter's height and body mass.

\subsection{Statistical analysis}

The mean and standard deviation (SD) were calculated for all variables. To analyze the relationship between the variables and the maximal height of the CG and the strain elastic energy, Pearson's productmoment correlation coefficient ( $r$ ) was used after performing the normality test of all variables, and the 90\% confidence intervals (CIs) were calculated. Furthermore, the magnitude of the correlation coefficients was assessed based on the following definition (Hopkins et al., 2009): $r<0.10$, trivial; $0.10 \leq r<0.29$, small; $0.30 \leq \mathrm{r}<0.49$, moderate; $0.50 \leq \mathrm{r}<0.69$, large; $0.70 \leq \mathrm{r}<0.89$, very large; $0.90 \leq \mathrm{r}<0.99$, nearly perfect; and perfect $=1$. Only the variables with $0.70 \leq \mathrm{r}$ were discussed as desirable indicators to evaluate the ability to bend the pole and the mechanical factors that can influence pole bending. All statistical analyses were performed using the SPSS software for Windows (version 25.0; International Business Machines Corp., New York, USA.).

\section{Results}

\subsection{Relationships between the maximal height of the CG and the indicators to evaluate bending of the pole}

The maximal height of the CG of the participants was $5.03 \pm 0.29 \mathrm{~m}$ (range: $4.46 \mathrm{~m}$ to $5.39 \mathrm{~m}$ ).

The time courses of $E_{p o l e}, E_{C F}$, and $E_{B M}$ were shown in Figure 1. The time courses of these variables were described as a bell-shaped curve from TO to PS. $E_{\text {pole }}$, $E_{C F}$, and $E_{B M}$ at MPB were $19.03 \pm 4.24 \mathrm{~J} / \mathrm{kg}$ (range: $11.79 \mathrm{~J} / \mathrm{kg}$ to $26.25 \mathrm{~J} / \mathrm{kg}$ ), $16.52 \pm 3.75 \mathrm{~J} / \mathrm{kg}$ (range: 
$10.21 \mathrm{~J} / \mathrm{kg}$ to $23.22 \mathrm{~J} / \mathrm{kg}$ ), and $2.52 \pm 0.74 \mathrm{~J} / \mathrm{kg}(1.57 \mathrm{~J} /$ $\mathrm{kg}$ to $3.67 \mathrm{~J} / \mathrm{kg}$ ), respectively.

The time courses of the bending rate and $A_{P B}$ were similar to the time course of $E_{C F}$ because $E_{C F}$ and bending rate were calculated based on $A_{P B}$. The maximal bending rate and the maximal $A_{P B}$ were $28.10 \pm$ $3.84 \%$ (range: $21.87 \%$ to $33.76 \%$ ) and $1.30 \pm 0.21 \mathrm{~m}$ (range: $0.95 \mathrm{~m}$ to $1.61 \mathrm{~m}$ ), respectively (Figure 2). The maximal bending rate and the maximal $A_{P B}$ showed large correlations with the maximal height of the $\mathrm{CG}(\mathrm{r}=0.86$ and 0.87 , respectively; Figure 2). By contrast, $E_{\text {pole }}$ at MPB showed a very large correlation with the maximal height of the CG $(r=0.94$; Figure 2).

\subsection{Relationship between the strain elastic energy and the mechanical energy}

The time courses of the vaulter's energy were shown in Figure 1. $E_{p}$ increased continuously through the vault. $E_{t}$ decreased from TO to MPB and increased from MPB to around PS. By contrast, $E_{r}$ was much smaller than $E_{p}$ and $E_{t}$. The relationships between $E_{\text {pole }}$ at MPB and the vaulter's energetic variables at TO and MPB were shown in Table 1. $E_{m}$ and $E_{t}$ at TO showed large correlations with $E_{\text {pole }}$ at MPB (r $=0.87$ and 0.88 , respectively). Moreover, $E_{m}$ and $E_{p}$ at MPB showed large correlations with $E_{\text {pole }}$ at MPB (r $=-0.73$ and -0.83 , respectively). The relationships between $E_{\text {pole }}$ at MPB and the amount of change in the vaulter's energetic variables from TO to MPB were shown in Table 1. $\Delta E_{p}$ and $\Delta E_{t}$ showed large correlations with $E_{\text {pole }}$ at MPB ( $\mathrm{r}=-0.80$ and -0.87 , respectively). On the contrary, $\Delta E_{m}$ showed a very large correlation with $E_{\text {pole }}$ at MPB $(\mathrm{r}=-0.91)$.

\subsection{Relationships between the strain elastic energy and the vaulting motions}

The time courses of the angular momentum and the
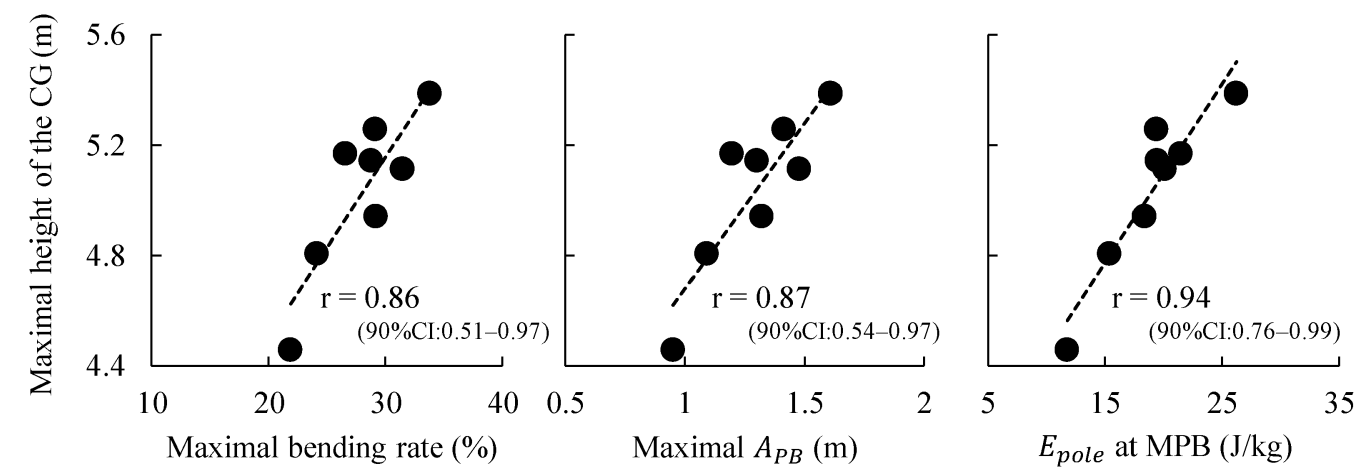

Figure 2 Relationships between maximal height of the $\mathrm{CG}$ and maximal bending rate, maximal $A_{P B}$, and $E_{p o l e}$ at MPB

Table 1 Relationships between $E_{\text {pole }}$ at MPB and mechanical energy of vaulter

\begin{tabular}{|c|c|c|c|c|c|c|c|c|c|c|c|c|}
\hline $\begin{array}{c}\text { Participant } \\
\text { (Maximal height } \\
\text { of the CG) }\end{array}$ & $\begin{array}{c}E_{m} \text { at TO } \\
(\mathrm{J} / \mathbf{k g})\end{array}$ & $\begin{array}{c}E_{p} \text { at TO } \\
(\mathrm{J} / \mathrm{kg})\end{array}$ & $\begin{array}{c}E_{t} \text { at TO } \\
(\mathrm{J} / \mathbf{k g})\end{array}$ & $\begin{array}{c}E_{r} \text { at TO } \\
(\mathrm{J} / \mathbf{k g})\end{array}$ & $\underset{(J / k g)}{E_{m} \text { at MPB }}$ & $\underset{(\mathrm{J} / \mathrm{kg})}{E_{p} \text { at MPB }}$ & $\begin{array}{c}E_{t} \text { at MPB } \\
(\mathrm{J} / \mathrm{kg})\end{array}$ & $\begin{array}{c}E_{r} \text { at MPB } \\
(\mathrm{J} / \mathrm{kg})\end{array}$ & $\begin{array}{c}\Delta E_{m} \\
(\mathbf{J} / \mathbf{k g})\end{array}$ & $\begin{array}{c}\Delta E_{p} \\
(\mathrm{~J} / \mathbf{k g})\end{array}$ & $\begin{array}{c}\Delta E_{t} \\
(\mathbf{J} / \mathbf{k g})\end{array}$ & $\begin{array}{c}\Delta E_{r} \\
(\mathbf{J} / \mathbf{k g})\end{array}$ \\
\hline A (5m39) & 48.66 & 11.95 & 36.18 & 0.54 & 27.09 & 21.18 & 5.62 & 0.29 & -21.58 & 9.23 & -30.56 & -0.25 \\
\hline $\mathrm{B}(5 \mathrm{~m} 26)$ & 40.27 & 12.32 & 27.07 & 0.87 & 29.36 & 23.65 & 5.45 & 0.25 & -10.91 & 11.34 & -21.62 & -0.62 \\
\hline $\mathrm{C}(5 \mathrm{~m} 17)$ & 39.18 & 12.30 & 26.13 & 0.74 & 27.95 & 22.37 & 5.26 & 0.33 & -11.22 & 10.07 & -20.87 & -0.42 \\
\hline $\mathrm{D}(5 \mathrm{~m} 14)$ & 44.78 & 12.06 & 32.07 & 0.65 & 29.10 & 23.28 & 5.49 & 0.33 & -15.68 & 11.22 & -26.58 & -0.31 \\
\hline $\mathrm{E}(5 \mathrm{~m} 11)$ & 41.86 & 12.13 & 29.40 & 0.34 & 27.88 & 23.72 & 3.92 & 0.23 & -13.99 & 11.60 & -25.48 & -0.11 \\
\hline $\mathrm{F}(4 \mathrm{~m} 94)$ & 42.65 & 11.93 & 29.28 & 1.44 & 28.41 & 22.16 & 5.99 & 0.25 & -14.24 & 10.24 & -23.29 & -1.19 \\
\hline $\mathrm{G}(4 \mathrm{~m} 81)$ & 37.85 & 12.78 & 24.63 & 0.44 & 29.33 & 24.04 & 4.96 & 0.33 & -8.53 & 11.26 & -19.67 & -0.12 \\
\hline $\mathrm{H}(4 \mathrm{~m} 46)$ & 33.51 & 12.22 & 20.94 & 0.35 & 28.80 & 24.48 & 4.05 & 0.27 & -4.71 & 12.25 & -16.88 & -0.08 \\
\hline Mean & 41.09 & 12.21 & 28.21 & 0.67 & 28.49 & 23.11 & 5.09 & 0.28 & -12.61 & 10.90 & -23.12 & -0.39 \\
\hline SD & 4.57 & 0.28 & 4.66 & 0.36 & 0.81 & 1.11 & 0.74 & 0.04 & 5.05 & 0.98 & 4.33 & 0.37 \\
\hline $\begin{array}{c}\mathrm{r} \\
(90 \% \mathrm{Cl})\end{array}$ & $\begin{array}{c}0.87^{*} \\
(0.57-0.97)\end{array}$ & $\begin{array}{c}-0.49 \\
(-0.85-0.20)\end{array}$ & $\begin{array}{c}0.88 * \\
(0.57-0.97)\end{array}$ & $\begin{array}{c}0.09 \\
(-0.57-0.68)\end{array}$ & $\begin{array}{c}-0.73 * \\
(-0.93--0.19)\end{array}$ & $\begin{array}{c}-0.83 * \\
(-0.96--0.42)\end{array}$ & $\begin{array}{c}0.43 \\
(-0.27-0.83)\end{array}$ & $\begin{array}{c}0.04 \\
(-0.60-0.65)\end{array}$ & $\begin{array}{c}-0.91 * \\
(-0.98--0.66)\end{array}$ & $\begin{array}{c}-0.80 * \\
(-0.85--0.35)\end{array}$ & $\begin{array}{c}-0.87^{*} \\
(-0.97--0.54)\end{array}$ & $\begin{array}{c}-0.09 \\
(-0.68-0.57)\end{array}$ \\
\hline
\end{tabular}


moment of inertia around the CG on each participant were shown in Figure 3. The angular momentum around the CG continued to decrease from TO to SW and then increased after SW. The moment of inertia around the CG reached the positive peak value at SW and decreased after that. Thereafter, the moment of inertia continued to decrease until MPB and increased after that.

The time courses of the angular momentum and the moment of inertia around the upper grip on each participant were shown in Figure 4. The angular momentum around the upper grip reached the negative peak value immediately after TO on all participants, and the positive peak value at MPB in almost all participants except for participant $\mathrm{H}$. For participant $\mathrm{H}$, the positive peak value of the angular momentum appeared before SW. The moment of inertia around the upper grip reached the positive peak value at SW and then decreased after that.

The relationships between $E_{\text {pole }}$ at $\mathrm{MPB}$ and the angular momentum and the moment of inertia were shown in Table 2. For variables around the CG, the negative peak value of angular momentum, the moment of inertia at SW, the maximal value of moment of inertia, and the average value of the angular mo- mentum from $\mathrm{TO}$ to $\mathrm{SW}$ showed large correlations with $E_{\text {pole }}$ at MPB ( $\mathrm{r}=-0.71,0.75,-0.81$, and -0.83 , respectively). On the other hand, for variables around the upper grip, the moment of inertia at $\mathrm{SW}$ and the average value of angular momentum from TO to SW showed large correlations with $E_{\text {pole }}$ at MPB (r $=0.79$ and -0.78 , respectively).

\section{Discussion}

Previous studies showed that the grip height has a significant effect on the vaulter's performance (Angulo-Kinzler et al., 1994; McGinnis, 1987). In addition, the availability of higher grip can be facilitated by bending the pole. Therefore, bending of the pole would affect the maximal height of the CG. On the other hand, a smaller force is required to bend the pole when the upper grip is closer to the upper tip of the pole (Euler's buckling load). Thus, the increase in the grip height is highly effective for large bending of the pole. However, there is a possibility of breaking the pole if it is bent too much; therefore, it is necessary to increase the stiffness of the pole to achieve a higher grip height while ensuring the recoil of the pole. Therefore, poles of various lengths and stiffness
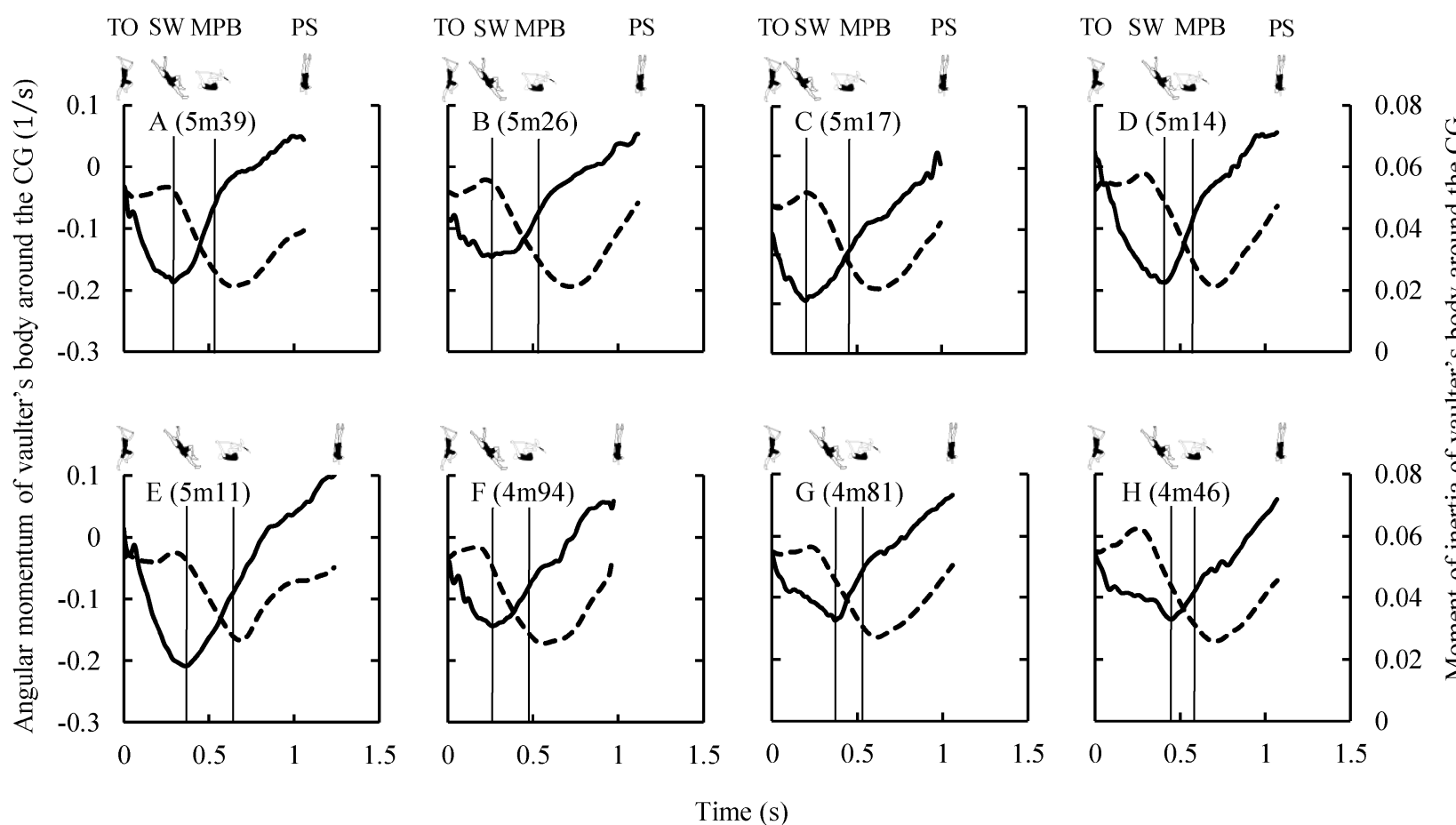

Time (s)

Figure 3 Time course of moment of inertia and angular momentum of vaulter's body around the CG Maximal height of the CG that each participant recorded is shown in ( ) 

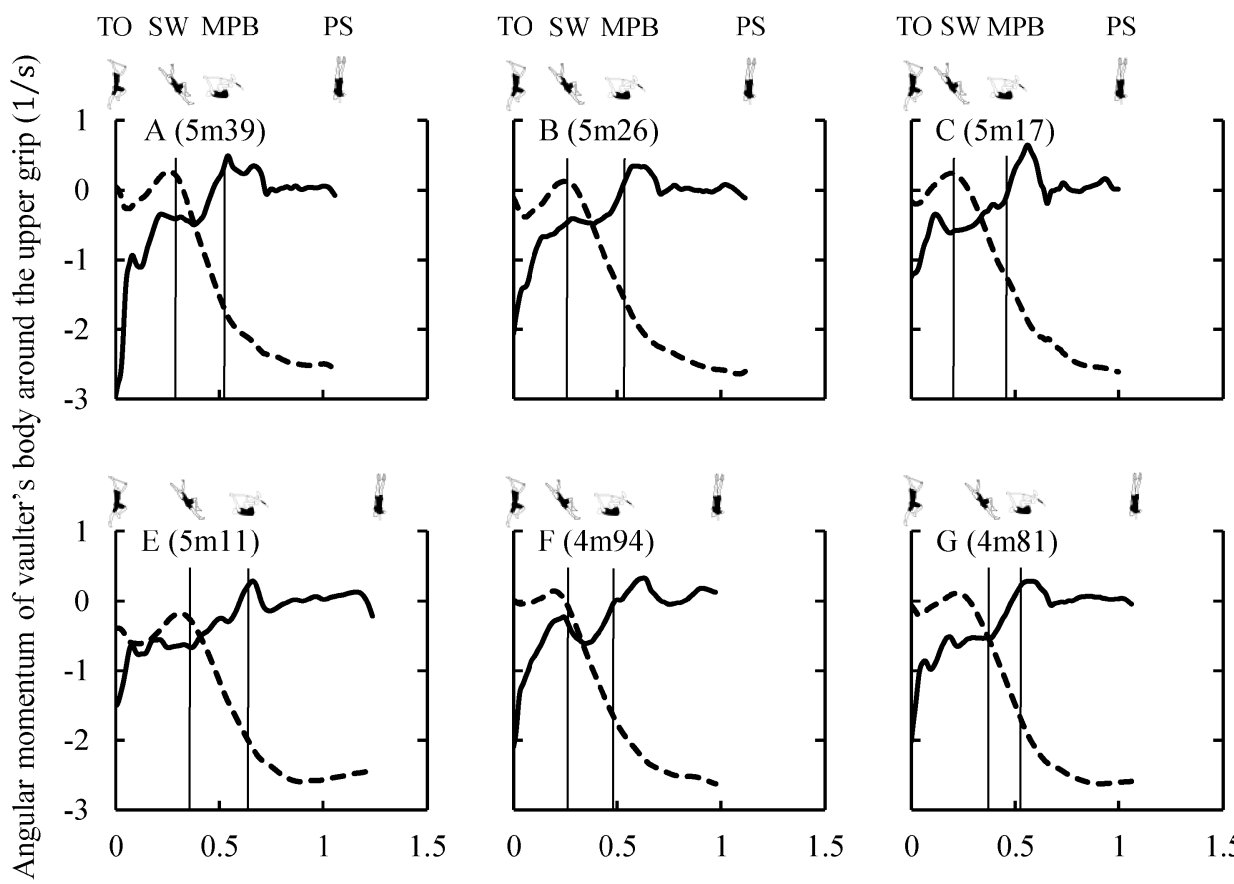

TO SW MPB PS
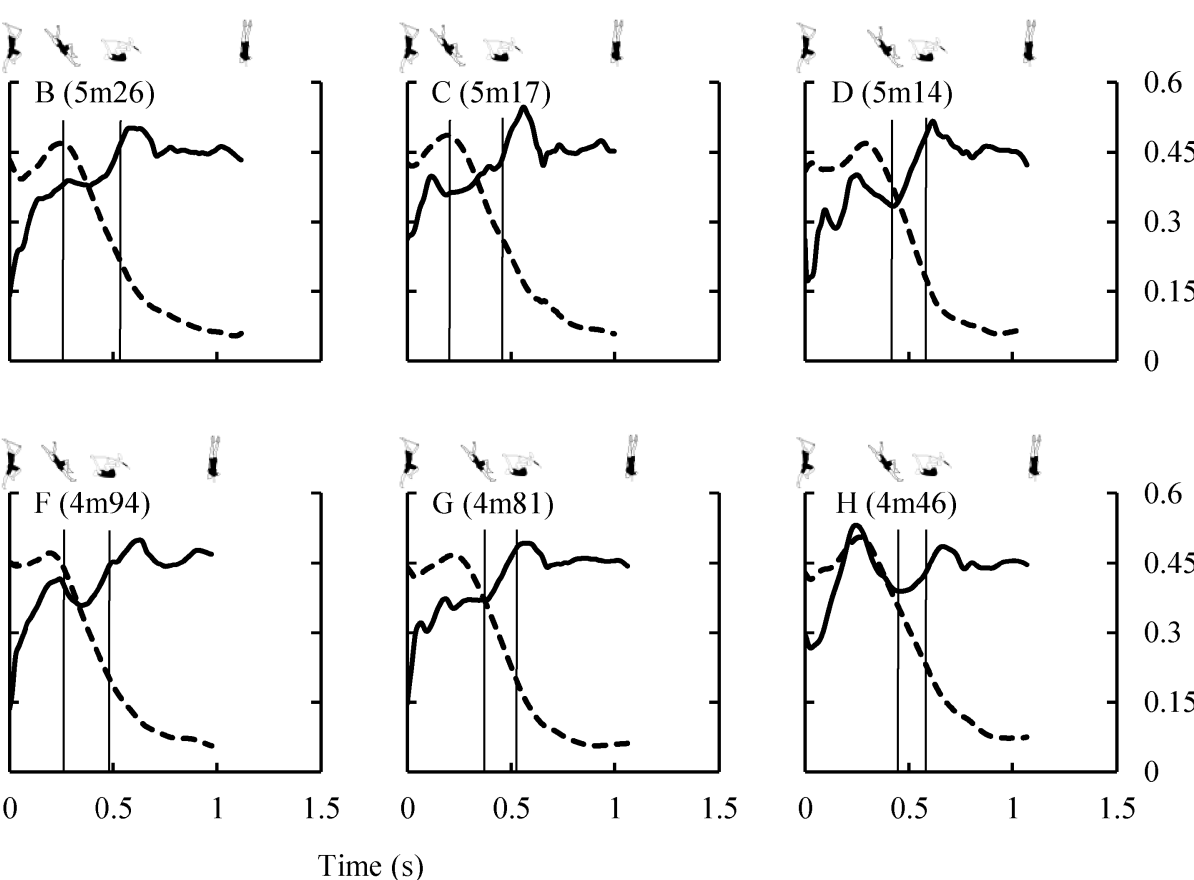

Time (s)

Figure 4 Time course of moment of inertia and angular momentum of vaulter's body around the upper grip

Table 2 Relationships between $E_{\text {pole }}$ at MPB and angular momentum and moment of inertia represent penetration and swing motion

\begin{tabular}{lcccc}
\hline \multicolumn{1}{c}{ Variables } & Mean & $\pm \mathrm{SD}$ & $\begin{array}{c}\text { r } \\
(90 \% \mathrm{CI})\end{array}$ \\
& & & & \\
\hline Around CG & -0.17 & \pm 0.03 & $-0.71^{*}(-0.93--0.15)$ \\
Negative peak value of angular momentum $(1 / s)$ & 50.15 & \pm 3.82 & $0.75^{*}(0.23-0.94)$ \\
Moment of inertia at $\mathrm{SW}\left(\times 10^{-3}\right)$ & 56.23 & \pm 3.12 & $-0.81^{*}(-0.95--0.37)$ \\
Maximal value of moment of inertia $\left(\times 10^{-3}\right)$ & -0.12 & \pm 0.02 & $-0.83^{*}(-0.96--0.42)$ \\
Average value of angular momentum from TO to SW $(1 / s)$ & 53.43 & \pm 2.31 & $-0.67(-0.91--0.08)$ \\
Average value of moment of inertia from TO to SW $\left(\times 10^{-3}\right)$ & & & \\
Around upper grip & -0.52 & \pm 0.14 & $-0.60(-0.89-0.04)$ \\
Angular momentum at SW $(1 / s)$ & 0.43 & \pm 0.05 & $0.79^{*}(0.32-0.95)$ \\
Moment of inertia at $\mathrm{SW}$ & 0.47 & \pm 0.02 & $-0.15(-0.71-0.53)$ \\
Maximal value of moment of inertia & -0.75 & \pm 0.22 & $-0.78^{*}(-0.94--0.30)$ \\
Average value of angular momentum from TO to SW $(1 / s)$ & 0.44 & \pm 0.02 & $-0.05(-0.66-0.60)$ \\
\hline Average value of moment of inertia from TO to SW & &
\end{tabular}

$*:|r|>0.7$

are used by each vaulter. Hence, the strain elastic energy in the pole was calculated in this study to take not only the length of the pole but also its stiffness.
Consequently, $E_{\text {pole }}$ at MPB showed higher correlation with the maximal height of the $\mathrm{CG}$ than the maximal bending rate and the maximal $A_{P B}$ (Figure 2). In other 
words, both the amount of bending of the pole and the stiffness of the pole need to be considered to evaluate the characteristics of the pole property and to optimize the grip height and final vault height. Meanwhile, it has been shown that the energy recovered from the pole to vaulter is $6-10 \%$ lower than $E_{\text {pole }}$ at MPB by the hysteresis of the pole (Arampatzis et al., 2004). However, it is difficult to extract only the recoiling force of the pole from the box reaction force including the vaulter's muscular work. In addition, it has been suggested that the influence of the energy loss on the vault is very small (Schade et al., 2006). Accordingly, present study disregarded the hysteresis of the pole.

Henceforth, the strain elastic energy in the pole will be discussed in detail. All participants took advantage of the interaction between the mechanical energy and the elastic energy in the pole during the vault (Figure 1 and Table 1). This phenomenon was similar to the results reported in previous studies (Arampatzis et al., 2004; Schade et al., 2000, 2004a, 2006; Schade et al., 2004b; Schade and Brüggemann, 2006; Sheehan, 2002). In addition, the compression force contributed significantly to the storage of the strain elastic energy (Figure 1). It has been clarified that the horizontal component of the box reaction force was larger than the vertical component of the box reaction force at the beginning of the vault (Frère et al., 2010). Thus, the storage of the strain elastic energy in the pole would be generated by conversion of $E_{t}$. Consequently, $\Delta E_{t}$ showed a large negative correlation with $E_{\text {pole }}$ at MPB (Table 1). On the other hand, it would be necessary to decrease the amount of $E_{t}$ when the vaulter drives horizontally after TO because the conversion from $E_{t}$ to $E_{p}$ is triggered when the vaulter jumps upward. As a result, $\Delta E_{p}$ showed a large negative correlation with $E_{\text {pole }}$ at MPB (Table 1). Therefore, the vaulter's motion that led to smaller increase in $E_{p}$ and larger decrease in $E_{t}$ from TO to MPB affected the storage of the strain elastic energy in the pole.

As mentioned earlier, the pole vault has been assumed as a double pendulum (Dyson, 1977; Hay, 1993). In present study, the angular momentum and the moment of inertia around the upper grip and the CG were calculated to represent the swing motion (Figure 3 and 4). As a result, these variables showed time courses similar to these reported in previous studies (Angulo-Kinzler et al., 1994; Gros and Kunkel, 1990). A vigorous swing facilitates larger bending of the pole because the reaction of the centripetal force acts on the upper grip (Hay, 1993). The magnitude of the centripetal force is obtained by combining the body's mass, the length of the radius and the body's angular velocity around the center of rotation. Accordingly, a quick swing with the body in extended posture would contribute to the storage of higher strain elastic energy in the pole. Consequently, the lower the angular momentum around the upper grip and the $\mathrm{CG}$ from $\mathrm{TO}$ to $\mathrm{SW}$, and the larger the moment of inertia around the upper grip and the CG at $\mathrm{SW}$, the higher $E_{\text {pole }}$ at MPB (Table 2). Thus, these results imply that the quick swing with the body in an extended position is effective for storage of the strain elastic energy in the pole because the vaulter could greatly apply the reaction of the centripetal force to the pole.

In order to store the higher strain elastic energy in the pole, it is necessary to act the larger force on the pole. On the other hand, the large box reaction force causes the rotation of the body around the upper grip and the CG because the force acts on the body through the upper grip and because of the distance between the upper grip and the CG. If the vaulter rotates immediately after TO, namely, a passive swing occurs, it will not likely complete until bar clearance because the velocity vector of the CG would turn upward earlier. In contrast, there is a possibility that a passive swing has not been occurred in this study because all participants completed vaults until bar clearance. Accordingly, it is inferred that there is a motion to avoid a passive swing, such as keeping a large moment of inertia around the upper grip and the CG after TO by fully extending the body. However, the moment of inertia around the CG and the upper grip from TO to SW did not show significant correlations with $E_{\text {pole }}$ at MPB (Table 2). The maximum and minimum values of moment of inertia around the CG were similar to the result reported in previous studies that analyzed the official performances of the world top pole vaulters (Angulo-Kinzler et al., 1994; Gros and Kunkel, 1990). Thus, these results imply that there is no difference in extension of their body after TO. Therefore, a vigorous swing with the body in a fully extended posture, as an opportunity to penetrate with a moderately extended posture after $\mathrm{TO}$, allows the storage of a larger strain elastic energy in the pole.

In this study, there is a possibility that a passive swing would not have occurred even though the degree of body extension was similar on all participants. 
Accordingly, there is a possibility that the vaulter has performed local body motions, such as shoulder joint flexion-extension motion, to avoid a passive swing. Meanwhile, it is important that the strain elastic energy is converted into mechanical energy again to achieve the higher maximal height of the CG. Previous studies suggested that the muscular work of the shoulder joint is important (Arampatzis et al, 1999; Arampatzis et al., 2004; Hubbard, 1980; McGinnis and Bergman, 1986). However, the motion of vaulter's upper body has not been clarified in detail biomechanically. Therefore, further studies should conduct three-dimensional analysis in order to clarify the vaulter's local motion that can influence the conversion from elastic energy to potential energy.

\section{Conclusion}

This study showed that the strain elastic energy in the pole can evaluate the ability to bend the pole more effectively than the bending rate and the amount of pole bending. Namely, bending of long and stiff poles enabled the vaulter to increase the available grip height and led to a corresponding increase in the maximal height of the vaulter's center of gravity. In addition, the smaller change in the potential energy and the larger change in the translational energy from take-off to maximal pole bending were effective for the storage of the higher strain elastic energy in the pole. Additionally, after focusing this study on "penetration" and "swing" as vaulter's motion, it was found that the vigorous swing with a fully extended posture, as an opportunity to penetrate with a moderately extended posture, was effective for storing strain elastic energy in the pole.

\section{References}

Angulo-Kinzler, R. M., Kinzler, S. B., Balius, X., Turro, C., Caubet, J. M., Escoda, J., and Prat, J. A. (1994). Biomechanical analysis of the pole vault event. J. Appl. Biomech., 10: 147165.

Arampatzis, A., Schade, F., and Brüggemann, G. P. (1999). Pole vault. Biomechanical Research Project at the VI ${ }^{\text {th }}$ World Championships in Athletics, Athens 1997: Final report (pp. 145-160). Monaco: International Athletic Foundation.

Arampatzis, A., Schade, F., and Brüggemann, G.-P. (2004). Effect of the pole-human body interaction on pole vaulting performance. J. Biomech., 37: 1353-1360.

Dillman, C. J. and Nelson, R. C. (1968). The mechanical energy transformations of pole vaulting with a fiberglass pole. J. Biomech., 1: 175-183.

Dyson, G. H. G. (1977). The mechanics of athletics (Seventh edition). New York: Homles and Meier Publishers.
Frère, J., Göpfert, B., Hug, H., Slawinski, J., and Tourny-Chollet, C. (2012a). Catapult effect in pole vaulting: Is muscle coordination determinant?. J. Electromyogr. Kines., 22: 145-152.

Frère, J., Göpfert, B., Slawinski, J., and Tourny-Chollet, C. (2012b). Effect of the upper limbs muscles activity on the mechanical energy gain in pole vauling. J. Electromyogr. Kines., 22: $207-214$

Frère, J., L'Hermette, M., Slawinski, J., and Tourny-Chollet, C. (2010). Mechanics of pole vaulting: A review. Sport. Biomech., 9: $123-138$.

Gros, H. J. and Kunkel, V. (1990). Biomechanical analysis of the pole vault. In: Brüggemann, G.-P. et al. (Eds.), Scientific report on the second IAAF world championship in athletics (2nd ed). Monaco: International Athletic Foundation.

Gudelj, I., Babić, V., Milat, S., Ćavala, M., Zagorac, S., and Katić, R. (2015). Differences in some kinematic parameters between two qualitatively different groups of pole vaulters. Coll. Antropol., 39: 41-46.

Hay, J. G. (1971). Mechanical energy relationships in vaulting with a fiberglass pole. Ergonomics, 14: 437-448.

Hay, J. G. (1993). The biomechanics of sport techniques (4th ed). New Jersey: Prentice Hall.

Hopkins, W., Marshall, S. W., Batterham, A. M., and Hanin, J. (2009). Progressive statistics for studies in sports medicine and exercise science. Med. Sci. Sport. Exer., 41: 3-12.

Hubbard, M. (1980). Dynamics of the pole-vault. J. Biomech., 13: 965-976.

International Association of Athletics Federations (IAAF) (2017). IAAF Competition Rules 2018-2019. Monaco: International Association of Athletics Federations.

Linthorne, N. P. and Weetman, A. H. G. (2012). Effect of run-up velocity on performance, kinematics, and energy exchanges in the pole vault. J. Sport. Sci. Med., 11: 245-254.

McGinnis, P. M. and Bergman, L. A. (1986). An inverse dynamic analysis of the pole vault. J. Appl. Biomech., 2: 186-201.

McGinnis, P. M. (1987). Performance limiting factors in the pole vault. Med. Sci. Sport. Exer., 19: S18.

Morlier, J. and Cid, M. (1996). Three-dimensional analysis of the angular momentum of a pole-vaulter. J. Biomech., 29: 10851090.

Morlier, J. and Mesnard, M. (2007). Influence of the moment exerted by the athlete on the pole in pole-vaulting performance. J. Biomech, 40: 2261-2267.

Petrov, V. (2004). Pole vault-the state of the art. New Study in Athletics, 19: 23-32.

Schade, F., Arampatzis, A., and Brüggemann, G.-P. (2000). Influence of different approaches for calculating the athlete's mechanical energy on energetic parameters in the pole vault. J. Biomech., 33: 1263-1268.

Schade, F., Arampatzis, A., and Brüggemann, G.-P. (2004a). A new way of looking at the biomechanics of the pole vault. New Study in Athletics, 19: 33-42.

Schade, F., Arampatzis, A., and Brüggemann, G.-P. (2006). Reproducibility of energy parameters in the pole vault. J. Biomech., 39: 1464-1471.

Schade, F., Arampatzis, A., Brüuggemann, G.-P., and Komi, P. V. (2004b). Comparison of the men's and the women's pole vault at the 2000 Sydney Olympic Games. J. Sport. Sci., 22: 835-842.

Schade, F. and Arampatzis, A. (2012). Influence of pole plant time on the performance of a special jump and plant exercise in the pole vault. J. Biomech., 45: 1625-1631.

Schade, F. and Brüggemann, G.-P. (2006). The pole vault at the 2005 IAAF World Championships in Athletics: A preliminary 
report. New Study in Athletics, 21: 57-66.

Sheehan, D. P. (2002). Beyond the pole vault: catapult - the fourth jump. Sports Eng., 5: 201-205.

Wells, R. P. and Winter, D. A. (1980). Assessment of signal noise in the kinematics of normal, pathological and sporting gaits. Human Locomotion, 1: 36-41.

Winter, D. A. (1990). Biomechanics and Motor Control of Human Movement (2nd Ed). New York: Wiley-Interscience.

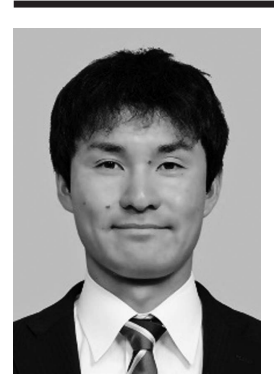

Name:

Takafumi Kageyuki

\section{Affiliation:}

${ }^{1}$ Graduate School of Comprehensive Human Sciences, University of Tsukuba

${ }^{2}$ Ibaraki Christian University

Address:

${ }^{1}$ 1-1-1 Tennodai, Tsukuba, Ibaraki 305-8574 Japan

26-11-1 Omikacho, Hitachi, Ibaraki 319-1295 Japan

Brief Biographical History:

Apr 2015- Mar 2017 Master's Program, Graduate School of Comprehensive Human Sciences, University of Tsukuba

Apr 2017- Doctoral Program, Graduate School of Comprehensive Human Sciences, University of Tsukuba

Apr 2020- Assistant, Ibaraki Christian University

Main Works:

-Kageyuki, T., Matsubayashi, T., Asai, T., Ohyama-Byun, K., and Kigoshi, K. (2020). Mechanical factors influencing maximal vertical velocity of body's center of gravity in pole vault. Japan Journal of Studies in Athletics, 16: 17-26. (in Japanese).

-Kageyuki, T., Matsubayashi, T., Asai, T., Ohyama-Byun, K., and Kigoshi, K. (2020). Relationship between bending of pole and motion of both grips in pole vault. Jpn J. Phys. Educ. Health Sport Sci, doi: 10.5432/jjpehss.19090. (in Japanese).

\section{Membership in Learned Societies:}

-International Society of Biomechanics in Sports

- Japan Society of Athletics

-Japan Society of Physical Education, Health and Sport Science

- Japanese Society of Biomechanics

- National Strength and Conditioning Association Japan

- The Japan Society of Coaching Studies 